\title{
Laparoscopic Roux-en-Y Gastric Bypass After Open Vertical Banded Gastroplasty in Patient with Severe GERD
}

\author{
Peter Vorwald ${ }^{1}$ - Rafael Mauricio Restrepo Núñez $^{2}$ - Maria Posada Gonzalez ${ }^{1}$. \\ Gabriel Salcedo Cabañas ${ }^{1}$
}

Published online: 7 March 2017

(C) Springer Science+Business Media New York 2017

\begin{abstract}
This video shows a laparoscopic reintervention after an open, vertical banded gastroplasty in a 39-year-old woman presenting with untreatable GERD.
\end{abstract}

Keywords Laparoscopic bypass Obesity · GERD

\section{Background}

The gastro-esophageal reflux disease (GERD) is an unwanted comorbidity after a vertical banded gastroplasty.

When the conservative treatment fails, the Roux-en-Y gastric bypass should be the procedure of choice.

\section{Materials and Method}

GERD was originated due to gastric remnant outlet obstruction, for that reason, we decided to perform a lap-

Electronic supplementary material The online version of this article (doi:10.1007/s11695-017-2626-5) contains supplementary material, which is available to authorized users.

Rafael Mauricio Restrepo Núñez

rafaelrestreponunez@gmail.com

1 Surgeon Esophagogastric unit Hospital Fundación Jiménez Díaz, Madrid, Spain

2 Surgery Resident Hospital Fundación Jiménez Díaz, Madrid, Spain aroscopic Roux-en-Y bypass. First, very intense adhesions of the greater omentum and the stomach to the parietal peritoneum and the left lobe of the liver are dissected. The gastric remnant is dissected in order to transect it proximal to the stenotic, banded segment. A Roux-en-Y gastric bypass with a $150-\mathrm{cm}$ alimentary limb using the OrVil ${ }^{\mathrm{TM}}$ orogastric tube and the DST Series $^{\text {TM }}$ EEA $^{\text {TM }} 25-\mathrm{mm}$ circular stapling device is performed.

\section{Results}

After 1 year from the surgery, the patient is asymptomatic.

\section{Conclusions}

The laparoscopic Roux-en-Y gastric bypass after an open banded vertical gastroplasty is a safe intervention, but it must be performed by experienced hands and in a hospital with bariatric surgery unit.

Compliance with Ethical Standards All procedures in studies involving human participants were in accordance with the ethical standards of the institutional and/or national research committee and with the 1964 Helsinki declaration and its later amendments or comparable ethical standards. Informed consent was obtained from all individual participants included.

Conflict of Interest The authors declare that they have no conflict of interest. 